\title{
CISTO INTRAESPINAL EM COMUNICAÇÃO COM O DISCO INTERVERTEBRAL NA COLUNA LOMBAR. RELATO DE UM CASO E REVISÃO DA LITERATURA
}

\author{
INTRASPINAL CYST COMMUNICATING WITH THE INTERVERTEBRAL DISK IN THE LUMBAR \\ SPINE. CASE REPORT AND LITERATURE REVIEW \\ QUISTE INTRAESPINHAL EN COMUNICACIÓN CON EL DISCO INTERVERTEBRAL EM LA \\ COLUMNA LUMBAR. REPORTE DE CASO Y REVISIÓN DE LA LITERATURA
}

\author{
Mauricio Marteleto Filho ${ }^{1}$, Paulo Henrioue Aguiar ${ }^{2}$, Marcos Vinicius Calfatt Maldaun ${ }^{2}$, Alexandros Theodoros Panagopooulos ${ }^{2}$, \\ Antonio Alberto Zambom³ ${ }^{3}$ Antonio Marcos de Souza Filho ${ }^{2}$, Nelson Paes Fortes Diniz Ferreira ${ }^{4}$, Pedro Augusto de Santana Junior ${ }^{5} \dagger$
}

\begin{abstract}
RESUMO
A hérnia discal lombar é causa importante bem como a mais freqüente de lombalgia e ciática. Muitos dos casos podem prontamente ser diagnosticados através do simples exame semiológico. A ressonância nuclear magnética (RNM) estabelece um diagnóstico definitivo mesmo em bases ambulatoriais. Diversos tipos de cistos intraespinais com diferentes patogenias foram descritos no passado como cistos perineurais, cistos sinoviais, cistos aracnóides e cistos ganglionares, que são doenças difíceis de diferenciar da hérnia de disco lombar. Os autores observaram recentemente um caso de cisto intraespinal comunicante com o disco intervertebral correspondente, apresentando sinais e sintomas e mesmo alguns achados radiológicos indistinguíveis daqueles da herniação discal lombar. O diagnóstico foi estabelecido pela RNM e confirmado no momento da cirurgia. Este artigo descreve as características dessa entidade clínica, incluindo quadro clínico, aparência radiográfica e achados histológicos, a fim de discutir possíveis patogenias e tratamento.
\end{abstract}

Descritores: Deslocamento do disco intervertebral; Dor lombar; Ciática; Cisto sinovial.

\begin{abstract}
Herniated lumbar disc is an important as well as the most common cause of low back pain and sciatica. Many cases can be readily diagnosed by simple semiological examination. Magnetic resonance imaging (MRI) provides a definitive diagnosis even in an outpatient basis. Several types of intraspinal cysts with different pathogenesis have been reported in the past, such as perineural cysts, synovial cysts, arachnoid cysts, and ganglion cysts, diseases that are difficult to differentiate from lumbar disc herniation. Recently, the authors have observed one case of intraspinal cyst communicating with the corresponding intervertebral disk, presenting clinical symptoms, signs, and even some radiographic findings undistinguishable from those of herniated disks. The diagnosis established by MRl and confirmed at surgery. This paper describes the features of this distinct clinical condition, including clinical findings, images, and histological aspects, to discuss possible pathogenesis and management.
\end{abstract}

Keywords: Intervertebral disk displacement; Low back pain; Sciatica; Synovial cyst.

\section{RESUMEN}

Una hernia de disco lumbar es una causa importante, así como la más frecuente causa del dolor lumbar y ciática. Muchos casos pueden ser fácilmente diagnosticados por el examen semiológico simple. La resonancia magnética (RM) proporciona un diagnóstico definitivo, incluso en atención ambulatoria. Varios tipos de quistes intraespinales con diferentes patogénesis se han descrito en el pasado como quistes perineurales, quistes sinoviales, los quistes aracnoideos y quistes ganglionares, enfermedades que son difíciles de diferenciar de la hernia de disco lumbar. Los autores observaron recientemente un caso de quiste intraespinal, que en comunicación con el disco intervertebral correspondiente, presentando signos y síntomas e incluso algunos hallazgos radiológicos que no se distinguen de los de la hernia discal lumbar. El diagnóstico fue determinado por RM y confirmado en la cirugía. En este artículo se describen las características de esta entidad clínica, incluyendo el aspecto clínico, radiológico e histológico, para discutir la posible patogénesis y tratamiento.

Descriptores: Desplazamiento del disco intervertebral, dolor de la región lumbar, ciática, quiste sinovial.

\section{INTRODUÇÃO}

Pareceu-nos bastante mais adequada a terminologia de "cisto intra espinal extradural" no lugar de "cisto pré-ganglionar", por circunscrever topograficamente a lesão, sem estabelecer vinculação etiológica. Ao mesmo tempo proporciona uma interpretação cirúr- gica investigativa da doença, abrangendo mais de uma etiologia, emprestando por si só à classificação um caráter patogenético, como veremos nas considerações finais sobre os eventos relacionados à sua formação.

O que veremos a seguir é o relato de um caso de cisto intra

1. Ortopedista do Hospital Santa Paula e Hospital Santa Catarina - São Paulo, SP, Brasil.

2. Neurocirurgião do Hospital Santa Paula e Hospital Santa Catarina - São Paulo, SP, Brasil.

3. Neurologista do Hospital Santa Catarina, Médico assistente da Divisão de Neurologia do Hospital das Clínicas da FMUSP - São Paulo, SP, Brasil.

4. Radiologista da MEDIMAGEM da Real Benemérita Beneficência Portuguesa - São Paulo, SP, Brasil.

5. † Neurocirurgião do Hospital Santa Paula e Hospital Santa Catarina - São Paulo, SP, Brasil (in memorian). 
espinal lombar sintomático, cujos achados semiológicos mimetizam os achados frequentes de dor lombar e ciática provocados pelas hérnias discais lombares. A presença de uma nova entidade nosológica de baixa incidência e prevalência, normalmente benigna, a partir dos dados da literatura levantada, suscita uma nova série de questões que dizem respeito às causas e consequencias desta condição, nem sempre óbvias. No caso descrito procuramos por meios diversos, estabelecer parte da etiopatogenia da doença sem contudo chegarmos a uma conclusão definitiva sobre os achados histológicos. O que permeamos através da condução do caso clínico e dos achados histopatológicos, na discussão e na leitura da literatura realizada é uma certa perplexidade sobre achados anatomo-patológicos que em momento algum afetam o tratamento, mas em contrapartida, coloca em cheque a natureza singular das conjecturas e da experiência dos pesquisadores nos trabalhos lidos, cuja interpretação pode ser controversa. Por este mesmo motivo é um bom exercício descritivo em que se tenta uma explicação minimamente convincente, ainda assim, dependente da opinião dos autores.

A discussão envolvendo sinais e sintomas bastante prevalentes, contrasta com a pequena quantidade de séries de pacientes tratados nos trabalhos estudados (1, 2, até cinco casos operados). A partir da suspeição do diagnóstico, nem sempre presente nos laudos da RM, e a despeito de quão frequente possa ser a dor lombar e ciática em nosso meio, grande parte delas provocadas por hérnias discais, ainda assim, pouco é dito sobre o assunto e a presença de tal entidade nosológica, em outras palavras, pouco diagnosticadas, nos leva a perguntarmo-nos sobre e com o que de fato lidamos, na abordagem dos cistos intra espinais. Como suspeitar, diagnosticar, interpretar, classificar e tratar tal entidade; qual o prognóstico? No caso descrito, quisemos conduzir o caso de forma clinicamente satisfatória, antevendo no diagnóstico por imagem, uma forma não invasiva de suspeição e questionando exames invasivos como a discografia peri-operatória, presente na maior parte dos trabalhos publicados, a qual consideramos desnecessária à medida em que adquirimos conhecimento e experiência com o assunto.

\section{RELATO DE CASO}

Paciente do sexo masculino de 31 anos, sofrendo de lombalgia seguida de irradiação para o membro inferior esquerdo por período de 3 meses, foi examinado em ambulatório. Inicialmente, foi tentado tratamento conservador com melhora. Após 6 meses o paciente apresentou uma nova crise de dor e foi internado no Hospital Santa Catarina. O paciente descreveu uma forte dor irradiada para o membro inferior esquerdo com alterações sensoriais no dermátomo de L5 e motoras. Os reflexos aquileu e patelar não estavam diminuídos. O teste de elevação da perna reta estava presente (a 30 graus ). Observamos discreta fraqueza motora do extensor digitorum brevis (EDB ) e distúrbio sensitivo moderado na raiz nervosa de L5 a esquerda. Não foram detectados distúrbios funcionais intestinais ou vesicais. Os sintomas tendiam a ser agravados pela posição ortostática e marcha, entretanto não havia posição específica do tronco agravando substancialmente os sintomas. A dor aumentava com a tosse ou a manobra de valsalva. Os raios- $X$ lombo-sacrais mostraram esclerose do pedículo de L4 esquerdo (Figura 1).

A ressonância nuclear magnética mostrou uma lesão esférica com baixa intensidade de sinal nas imagens pesadas em T1 e alta intensidade de sinal nas imagens pesadas em T2 (Figuras 2 A e B). Foi submetido a procedimento cirúrgico e o cisto foi localizado imediatamene abaixo da raiz nervosa emergente de $L 4$ e acima da raiz nervosa de L5 onde foi separado do saco dural (Figura $3 \mathrm{~A}, \mathrm{~B}$ ). A adesão entre o cisto e a raiz nervosa era moderada (Figura 3 C, D ) e o cisto pode ser totalmente removido em pedaços devido a significativa hemorragia do corpo vertebral posterior e espaço epidural (Figura 3E ), entretanto o exato ponto de sangramento não pode ser precisamente identificado. Uma hemostasia foi obtida pela cuidadosa manipulação de materiais hemostáticos como a cola de fibrina e gelatina hemostática.

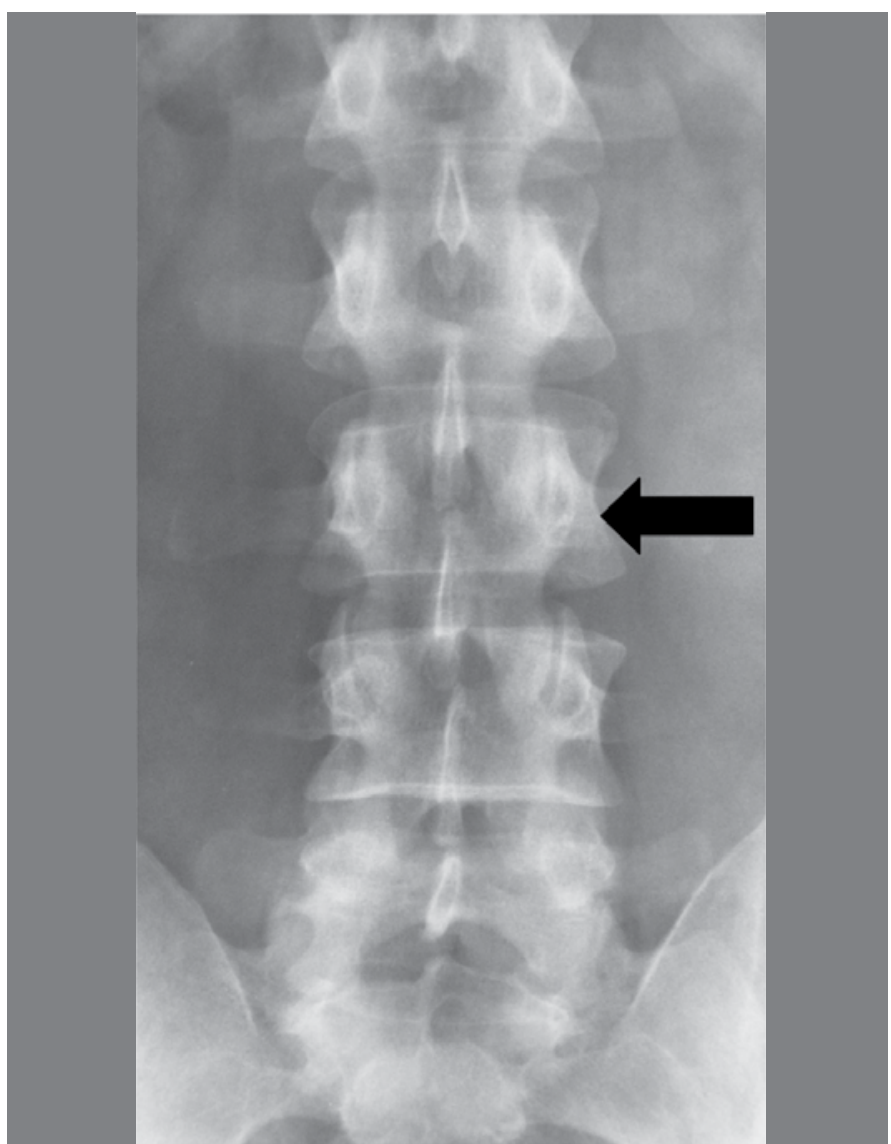

Figura 1. Raio-X mostrando esclerose no pedículo esquerdo de L4 ( seta )

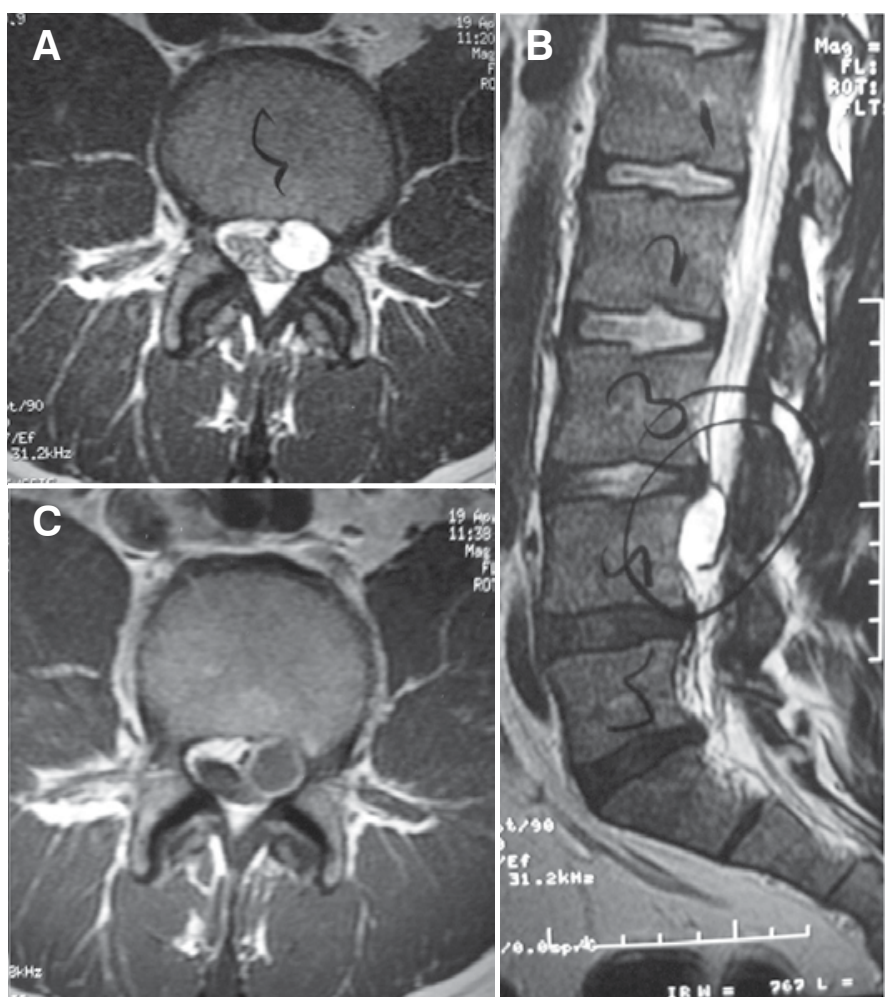

Figura 2- Imagens de ressonância nuclear magnética do paciente. A, B, imagens pesadas em T2. Um evidente cisto de formato oval com alto sinal de intensidade está presente por trás do corpo vertebral de L4. C, Contraste da imagem pesada em T1. A periferia da parede do cisto está claramente contrastada com ácido penta-acético diethylenotriamina-gadolínio 

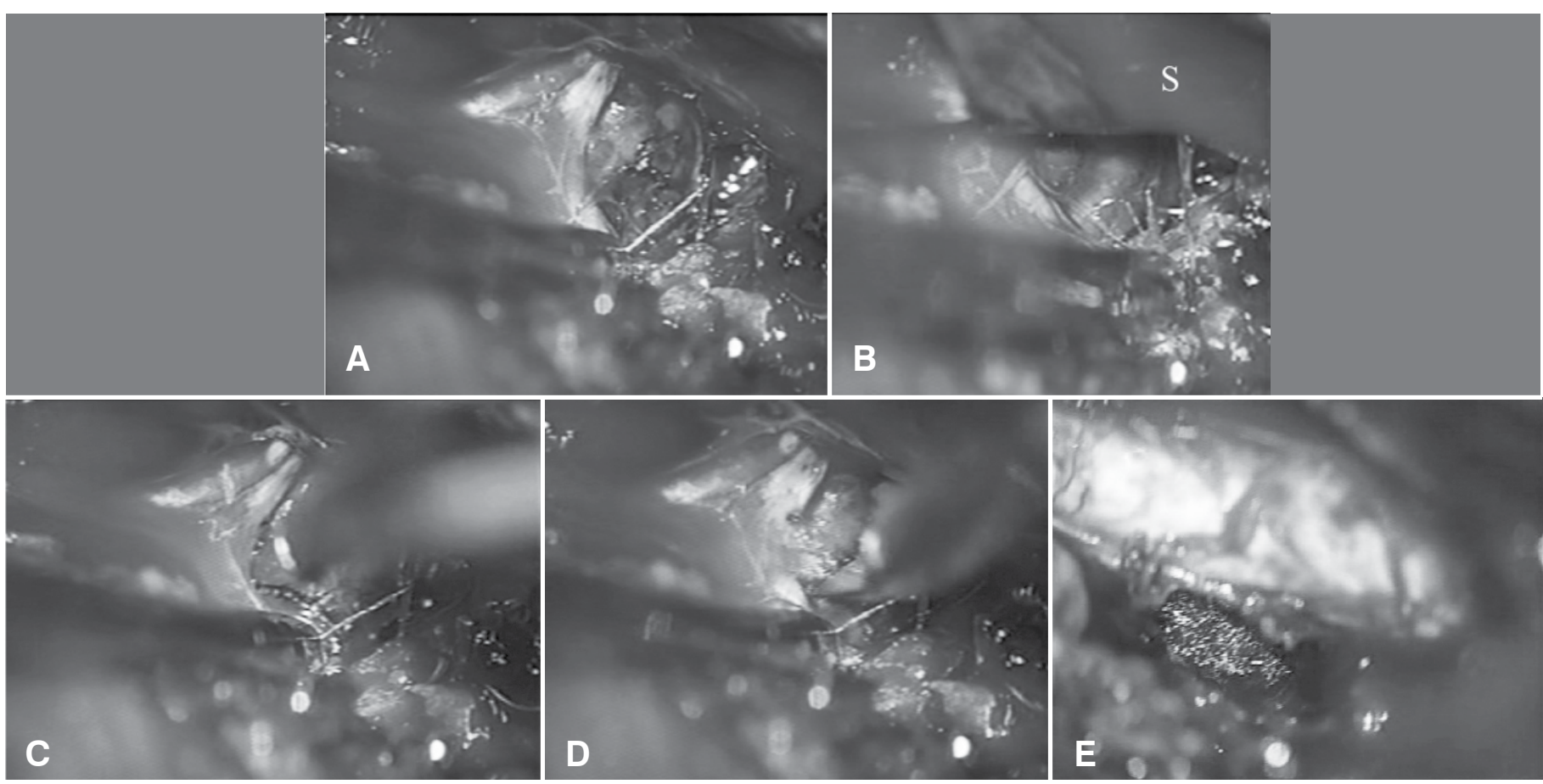

Figura 3- Imagens do ato intra-operatório mostrando a retirada do cisto. A e B; A raiz nervosa de L5 foi separada da parede do cisto. C e D; A adesão entre a raiz nervosa e o cisto era moderada. $\mathrm{E} ; \mathrm{O}$ cisto pode ser removido em pedaços devido à significativa hemorragia do corpo vertebral posterior.

O exame histológico realizado mostrou um denso tecido fibro-conectivo sem células de revestimento específicas, ausência de tecidos discais, tecido nervoso ou células tumorais.

O paciente teve alta hospitalar após o quarto dia pós-operatório (e) sem dor.

\section{DISCUSSÃO}

Muitos cistos intra-espinais podem mimetizar sinais e sintomas muito similares àqueles da herniação discal lombar, incluindo os cistos perineurais originados da bainha de mielina ${ }^{1,2}$, cistos sinoviais originados da faceta $\operatorname{articular}^{3}$ e cistos ganglionares ${ }^{4,5}$. Podem ser causados pela degeneração mucóide do tecido conectivo devido a cargas dinâmicas repetitivas exercidas na cápsula das facetas articulares, ou no ligamento longitudinal posterior ${ }^{4,5}$. Existem várias causas adicionais, como o cisto causado pela produção gasosa em discos extremamente degenerados ${ }^{6,7}$, cistos de ligamento amarelo ${ }^{8}$, varizes epidurais ${ }^{9}$ e hematomas pré-membranosos.

Os achados dos cistos intra-espinais descritos neste relato de caso pode ser mencionado da seguinte forma: sintomas clínicos indistinguíveis daqueles presentes em pacientes com uma típica herniação discal que se manifesta como lesão única da raiz nervosa unilateral, com incidência numa idade um pouco mais jovem e no nível discal intervertebral acima do que a típica herniação discal. Uma lesão circular a oval com intensidade de sinal baixa em T1 e alta em T2 compatível com um cisto mas não com uma herniação discal, degeneração mínima do disco envolvido em ambas discografia/TC discografia e RNM, conexão entre o cisto e os discos intervertebrais correspondentes nos discogramas, com reprodução da dor irradiada para a perna afetada no momento da injeção e bom resultado clínico incluindo alívio imediato da dor na perna pela simples remoção do cisto (a parede do cisto consiste de tecido conectivo fibroso denso de conteúdo hemático-seroso claro) e ausência de material discal ou células de revestimento específicas no exame histológico.

O cisto descrito pelos autores presentes é muito similar ao hematoma pré-membranoso descrito por Wiltse et al. ${ }^{10}$, varizes epidurais lombares (Tipo 3 ) descritas por Hanley et al. ${ }^{11}$ e mesmo alguns casos primariamente reportados como cistos ganglionares ${ }^{4,5}$.
Provavelmente, estes cistos possam ser desordens idênticas com nomes diferentes. Recentemente, diversos artigos foram publicados reportando cistos intra-espinais com uma comunicação patente com o disco intervertebral ${ }^{12-16}$.

A etiologia do cisto reportada no presente estudo não é clara. Muitas causas patogenéticas poderiam ser especuladas, como a reabsorção de uma hérnia discal pré-existente, hematoma associado ao prolapso discal ${ }^{10}$ e degeneração mucóide especial, sendo esta última reverenciada como a causa do cisto ganglionar no tecido peridiscal ${ }^{4}$

A parede do cisto é evidenciada pelo Gadolino na RNM, um achado similar ao evidenciamento frequentemente encontrado na periferia do disco herniado durante o processo de regressão espontânea da hérnia. Se estes cistos aumentam como conseqüência da reabsorção do material discal extruso, o disco correspondente deveria estar mais degenerado. Por outro lado, não foi possível achar mais do que degeneração discal moderada em ambos discogramas e na RNM. A intensidade de sinal do cisto foi completamente diferente daquela do disco herniado, e em um paciente que realizou RNMs seriadas, nenhum disco herniado foi encontrado na RNM obtida antes do desenvolvimento do cisto. Durante a cirurgia, a lesão tinha uma cápsula distinta composta de tecido conectivo fibroso de conteúdo líquido, e nenhum material discal foi encontrado dentro do cisto (Figuras $3 \mathrm{~A}, \mathrm{~B}$ e C). Os achados histológicos também foram diferentes daqueles encontrados nos discos herniados em regressão, como a ausência de neovascularização e infiltração celular, que são comumente encontrados em especimens dos pacientes com discos herniados ${ }^{17}$. Se levarmos em consideração estes fatos, o desenvolvimento do cisto é menos provavelmente atribuível a regressão espontânea de um prolapso discal.

A revisão da literatura nos mostra que a discografia e os achados cirúrgicos revelaram que o cisto era sempre localizado entre a linha média do corpo vertebral posterior e os pedículos. $\mathrm{Na}$ coluna lombar, o ligamento longitudinal posterior cobre o espaço discal e a porção central posterior dos corpos vertebrais ${ }^{10}$. Outras partes do corpo vertebral são cobertas pela membrana peridural somente $^{18,19}$. Onde existe ligamento longitudinal posterior, esta membrana existe entre o ligamento e o corpo vertebral. Vasos sanguíneos penetram esta membrana para formar uma densa rede 
anastomótica, por comunicação entre o plexo venoso peridural (plexo vertebral antero-medial ), que tem um rico fluxo sanguíneo, e as veias basi-vertebrais. Estas veias podem estar em risco quando ocorre uma hérniação discal, separando a membrana peridural dos corpos vertebrais e causando um hematoma abaixo da membrana peridural. Se estes cistos formados como conseqüência de um hematoma que se desenvolve no momento da herniação discal, como descrito por Wiltse et $a^{10}{ }^{10}$, a média temporal entre o início do sintoma inicial e os procedimentos cirúrgicos foi de 17 meses, e mesmo entre o início da dor na perna e os procedimentos cirúrgicos foram de 5 meses, ambos foram extremamente mais longos do que o período necessário para o processo de reabsorção normal de um hematoma ${ }^{20}$ ou seja, o hematoma deve ter sido resolvido bem antes do desenvolvimento do cisto. Além disso, nenhuma evidência de herniação discal foi encontrada quer seja radiograficamente ou intra-operatoriamente ${ }^{20}$, no paciente do presente estudo.

De acordo com os achados cirúrgicos, o conteúdo do cisto foi sero-sanguinolento de consistência mais espessa e nenhum coágulo sanguíneo foi encontrado. Portanto, a simples idéia de que o cisto tenha surgido de um hematoma associado a uma herniação prolapsada é ainda controversa.

Outra hipótese fala a favor de uma lesão discal inicial como base para o desenvolvimento do cisto. A Hemorragia do plexo peridural de alto fluxo ocorre mesmo sem a presença de um disco prolapsado como conseqüência da separação da membrana peridural do corpo vertebral causada por uma força mecânica transmitida por uma fissura no anel fibroso que se desenvolve no disco intervertebral posterior. Se for considerado que a lombalgia se desenvolve no anulo fibroso fissurado, e a ciática ocorre quando o cisto se forma, um mínimo de 0 meses e um máximo de 50 meses (média 6,4 meses) constituiria um período de tempo entre a ruptura do anulo fibroso e o desenvolvimento dos $\operatorname{cistos}^{20}$ o que difere da história natural destes casos em estudo.

Um mecanismo desconhecido que envolve a reabsorção do hematoma pode desempenhar um certo papel neste caso, como a degeneração mucosa na superfície do hematoma encapsulando-o e levando a formação tardia de um cisto ${ }^{21}$. Por outro lado, este cisto pode verdadeiramente ser um cisto ganglionar de origem no disco intervertebral e o seu mecanismo envolva sobrecarga axial imposta a um disco intervertebral moderadamente degenerado e sujeito a altas pressões internas. A carga transmitida através da fissura no anel fibroso em sua porção posterior criariam a lesão discal e o estresse cinético nas estruturas intra-espinais como as lesões do ligamento longitudinal posterior e na membrana peridural. Existe a possibilidade de que a sobrecarga axial possa desempenhar um papel no desenvolvimento dos cistos como é o caso dos cistos ganglionares em outras partes do corpo. De fato, todas as paredes de cistos examinadas no presente estudo eram constituídas de denso tecido fibro-conectivo sem células específicas de revestimento, entretanto, estas podem ser facilmente diferenciadas daquelas dos cistos sinoviais e perineurais, mas foram difíceis de diferenciar dos cistos ganglionares. Provavelmente muitos cistos ganglionares que foram reportados previamente poderiam ter achados discográficos similares aos do cisto descrito no presente estudo se os devidos estudos tivessem sido feitos nestes trabalhos.

Qualquer que seja a patogênese, o cisto intra-espinal fora responsável pelo aparecimento dos sintomas, não a herniação discal. Lesões do disco intervertebral subjascente e forças menores geradas por excessivo movimento da coluna podem ser responsáveis pelo desenvolvimento dos cistos, deixando, portanto uma comunicação com o disco correspondente detectadas em ambos os casos. Dado que o cisto descrito no presente estudo é similar aos cistos meniscais ${ }^{1}$, os quais se acredita serem causados pelo trauma da articulação do joelho ${ }^{1}$ e que estes cistos são de origem do disco intervertebral, presupõe-se para esta entidade clínica ser chamada de cisto discal.

No momento, a discografia ${ }^{22,23}$ é a única ferramenta que faz o diagnóstico pré-operatório dos cistos discais. Entretando, se a discografia é absolutamente necessária ou não em todos os pacientes com cistos intra-espinais é controverso ${ }^{23}$, porque a remoção dos cistos leva a melhora dos sintomas de forma independente de sua origem histo-genética ${ }^{24}$. Os autores acreditam que o acúmulo de mais informações clínicas possam promover o reconhecimento e o estabelecimento desta nova entidade clínica e fazer a discografia desnecessária como ferramenta diagnóstica num futuro próximo.

\section{REFERÊNCIAS}

1. Barrie HJ, et al. The pathogenesis and significance of menisceal cysts. J Bone Joint Surg Br. 1979:61(2):184-9.

2. Tarlov IM, et al. Perineural cysts of the nerve roots. Arch Neurol. 1938:40:1067-74.

3. Yuh WT, Drew JM, Weinstein JN, McGuire CW, Moore TE, Kathol MH, et al. Intraspinal synovial cysts. Magnetic resonance evaluation. Spine (Phila Pa 1976). 1991;16(7):740-5

4. Brish A, Payan HM, et al. Lumbar intraspinal extradural ganglion cyst. J Neurol Neurosurg Psychiatry. 1972;35(6):771-5

5. Kao CC, Uihlein A, BickelWH, Soule EH, et al. Lumbar intraspinal extradural ganglion cyst. J Neurosurg. 1968:29(2):168-72.

6. Hidalgo-Ovejero AM, Martinez-Grande M, Garcia-Mata S, et al. Disc herniation with gas. Spine (Phila Pa 1976). 1994;19(19):2210-2

7. Mortensen WW, Thorne RP, Donaldson WF $3^{\text {rd }}$, et al. Symptomatic gas-containing disc herniation. Report of four cases. Spine (Phila Pa 1976). 1991;16(2):190-2.

8. Baker JK, Hanson GW, et al. Cyst of the ligamentum flavum. Spine (Phila Pa 1976). 1994;19(9):1092-4

9. Goyal RN, Russell NA, Benoit BG, Belanger JM, et al. Intraspinal cysts: a classification and literature review. Spine (Phila Pa 1976). 1987;12(3):209-13.

10. Wiltse LL, Fonseca AS, Amster J, Dimartino P, Ravessoud FA, et al. Relationship of the dura, Hofmann's ligaments, Batson's plexus, and a fibrovascular membrane lying on the posterior surface of the vertebral bodies and attaching to the deep layer of the posterior longitudinal ligament. An anatomical, radiologic, and clinical study. Spine (Phila Pa 1976). 1993:18(8):1030-43.

11. Hanley EN Jr, Howard BH, Brigham CD, Chapman TM, Guilford WB, Coumas JM, et al Lumbar epidural varix as a cause of radiculopathy. Spine (Phila Pa 1976). 1994;19(18):2122-6.

12. Chatani $K$, Kamata $Y$, Shirokura M, et al. Intraspinal cystic hematoma as sociated with lumbar disc herniation. J Jpn Orthop Assoc. 1996;70:S316. [in Japanese]

13. Nishizawa T, Koyanagi T, Toyama Y, et al. Three cases of intraspinal cyst whose symptoms resembled lumbar disc herniation. Seikei Geka. 1995;46:1353-7.[in Japanese]

14. Tokutani S, KatanoH, Ichikawa S, Ishibashi K, Echigoya N, Miura K, Yokoyama T, Tomita S, et al.: [Intraspinal cyst (premembranous hematoma): a case report]. [in Japanese]

15. Nishizawa T, Koyanagi T, Toyama Y, et al. Three cases of intraspinal cyst whose symptoms resembled lumbar disc herniation. Seikei Geka 1995;46:1353-7 [in japanese].

16. Toyama $Y$, Kamata $M$, Matsumoto $M$, et al. Pathogenesis and diagnostic of intraspinal cyst communicating with intervertebral disk in the lumbar spine.Rinsho Seikei Geka. 1997;32:393-400. [in Japanese].

17. Yasuma T, Arai K, Yamauchi Y, et al. The histology of lumbar intervertebral disc herniation The significance of small blood vessels in the extruded tissue. Spine (Phila Pa 1976). 1993:18(13):1761-5

18. Dommisse GF, et al. The Arteries and veins of the human spinal cord from birth. Edinburgh: Churchill Livingstone; 1975

19. Kikuchi $\mathrm{S}$, et al. Anatomical and experimental studies of nerve root infiltration. Nihon Seikeigeka Gakkai Zasshi. 1982:56(7):605-14

20. Chiba K, Toyama Y, Matsumoto M, Maruiwa H, Watanabe M, Nishizawa T, et al. Intraspinal cyst communicating with the intervertebral disc in the lumbar spine: discal cyst. Spine (Phila Pa 1976). 2001;26(19):2112-8.

21. Fujimura Y, Wakano K, Hijikata S, et al. Diagnostic value of lumbar discog raphy. Seikeigeka 1974:25:781-90 [in Japanese].

22. Demierre B, Ramadan A, Hauser $H$, Reverdin A, Rilliet B, Berney J, et al. Radicular compression due to lumbar intraspinal gas pseudocyst: case report. Neurosurgery. 1988:22(4):731-3.

23. Gibson MJ, Buckley J, Mawhinney R, Mulholland RC, Worthington BS, et al. Magnetic resonance imaging and discography in the diagnosis of disc degeneration. A comparative study of 50 discs. J Bone Joint Surg Br. 1986:68(3):369-73.

24. McCulloch JA, Transfeld EE, et al. Disc degeneration with root irritation: disc ruptures. In: Macnab's backache. 3rd ed. Baltimore: Williams \& Wilkins; 1997. p. 500-68. 